typeset using JPSJ.sty $<$ ver.1.0b $>$

\title{
Thermal Properties of Heavy Fermion Compound YbP
}

\author{
Naoyuki TATEIWA*, Tatsuo C KOBAYASHI, Kiichi AMAYA ${ }^{1}$, Dexin LI², \\ Yoshinobu SHIOKAWA ${ }^{2}$ and Takashi SUZUKI ${ }^{3}$
}

\author{
Research Center for Materials Science at Extreme Conditions, Osaka University, Toyonaka, Osaka 560-8531, \\ Japan \\ ${ }^{1}$ Department of Physical Science, Grasuate School of Engineering Science, Osaka University, Toyonaka, Osaka \\ 560-8531, Japan \\ 2 Oarai Branch, Institute for Materials Research, Tohoku University, Oarai, Ibaraki, 311-1313, Japan \\ 3 Physics Department, Graduate School of Science, Tohoku University,Sendai 980-8545, Japan
}

(Received May 10, 2001 )

\begin{abstract}
Low-temperature specific heat and its field-dependence up to $16 \mathrm{~T}$ was measured in a stoichiometric single crystal of $\mathrm{YbP}$. A sharp peak was observed at $T_{\mathrm{N}}=0.53 \mathrm{~K}$ in zero magnetic field. Application of external field seems to induce a new magnetic phase above $11 \mathrm{~T}$. The field dependence of the transition temperature in the high-field phase is different from that of the low field phase. The linear coefficient of the electronic specific heat is estimated as $120 \mathrm{~mJ} / \mathrm{mole}^{2}$ from low temperature specfic heat, suggesting heavy Fermion state in YbP.
\end{abstract}

KEYWORDS: YbP, magnetic phase diagram, heavy-Fermion state

\section{§1. Introduction}

The $\mathrm{Yb}$ monopnictides $\mathrm{YbX}_{\mathrm{p}}\left(\mathrm{X}_{\mathrm{p}}=\mathrm{N}, \mathrm{P}\right.$, As and $\mathrm{Sb})$ with the cubic NaCl-type crystal structure are semimetallic compounds with an extremely low carrier concentration. ${ }^{1,2)}$ The $\mathrm{Yb}$ ion in $\mathrm{YbX}_{\mathrm{p}}$ is predominantly trivalent with one $4 f$ hole, in which the spin-orbit split $J=7 / 2$ is the ground state configuration and it further splits into $\Gamma_{6}, \Gamma_{8}$, and $\Gamma_{7}$ states in the cubic crystal field with $\Gamma_{6}$ doublet as the ground state. ${ }^{3-5)}$ The band calculation indicates that the bottom of the conduction band, formed mainly by $5 d(\mathrm{Yb})$, is at each $\mathrm{X}$ point, slightly overlapping with the top of the valence band to make semimetals with a low carrier concentration of the order of 0.01 per $\mathrm{Yb}^{3+}$ ion. ${ }^{6,7)}$ The $\mathrm{Yb}$ monopnictides have attracted a lot of attention in the last decade because of their various puzzling properties. At around $0.5 \mathrm{~K}$, the stoichiometric compounds $\mathrm{YbN}$ and $\mathrm{YbAs}$ show antiferromagnetic fcc type-III order, and nonstoichiomatric $\mathrm{YbP}_{0.84}$ undergoes a magnetic ordering corresponding to fcc type-II antiferromagnetism. ${ }^{8-10}$ ) The ordered magnetic moments in $\mathrm{YbN}, \mathrm{YbP}$ and $\mathrm{YbAs}$ are much smaller than the value of $1.33 \mu_{\mathrm{B}}$ expected from the crystalline-electric field $(\mathrm{CEF})$ ground state doublet $\Gamma_{6}$. The specific heat measurement shows a broad peak around $5 \mathrm{~K}$ and the entropy associated with the antiferromagnetic ordering are 20-30\% of $R \ln 2$ in $\mathrm{YbN}$, $\mathrm{YbP}$ and YbAs. Moreover, the coefficient of electronic specific heat $\gamma$ was estimated to be $270 \mathrm{~mJ} / \mathrm{moleK}^{2}$ for YbAs. ${ }^{11)}$ From these results, Yb monopnictides have been considered to be heavy-Fermion compounds with the Kondo effect. However, the - $\ln T$ behavior characteristic of Kondo compounds is not detected in the electri-

\footnotetext{
*E-mail:tateiwa@djebel.mp.es.osaka-u.ac.jp
}

cal resistivity of YbAs and YbP. There is not a theoretical model that could give a comprehensive interpretation of all the experimental results. Clearly, the anomalous physical properties in YbXp should be studied in more detail. In particular, experimental works on high quality single crystals are necessary.

It is very difficult to grow single crystals of $\mathrm{YbX}_{\mathrm{p}}$ due to the high melting point and a high vapor pressure. So far, single crystals of $\mathrm{YbX}_{\mathrm{p}}$ were prepared only for $\mathrm{YbAs}$ and $\mathrm{YbP}$, but the $\mathrm{YbP}$ sample was a nonstoichiometric one, ${ }^{12)}$ and thus intrinsic physical property, in particular transport properties, was not reported for $\mathrm{YbN}$, YbP and $\mathrm{YbSb}$ until now. Recently, Li et al. has succeeded in growing the stoichiometric single crystal of YbP. Their ex perimental results revealed the smaller residual resistivity $R R=\rho(T \rightarrow 0 \mathrm{~K})=6.9 \mu \Omega \mathrm{cm}$, the larger residual resistance ratio $R R R=\rho(T=300 \mathrm{~K}) / \rho(T \rightarrow$ $0 \mathrm{~K})=8.5$, and the larger positive magnetoresistance $M R=[\rho(H)-\rho(0)] / \rho(0)=1.23$ (at $H=10 \mathrm{~T}$ and $T$ $=4.2 \mathrm{~K})$. The corresponding values for nonstoichiometric $\mathrm{YbP}_{0.84}$ are $R R=17.2 \mu \Omega \mathrm{cm}, R R R=3$ and $M R=$ 0.08 . To our knowledge, this $\mathrm{YbP}$ single crystal is the best sample at present. In this paper, we present the result of specific heat measurement performed on this YbP single crystal under magnetic field up to $16 \mathrm{~T}$ applied along $\langle 100\rangle$ direction. A large coefficient of electric specific heat and a new magnetic phase under high magnetic field were detected in $\mathrm{YbP}$.

\section{§2. Experimental}

The single crystal sample used in this study was grown by the mineralization method in a tungsten crucible. The detailed description of the sample preparation is given in ref. 13. The powder sample was first prepared by prereacting starting elements with a composition of $\mathrm{Yb}: \mathrm{P}$ 


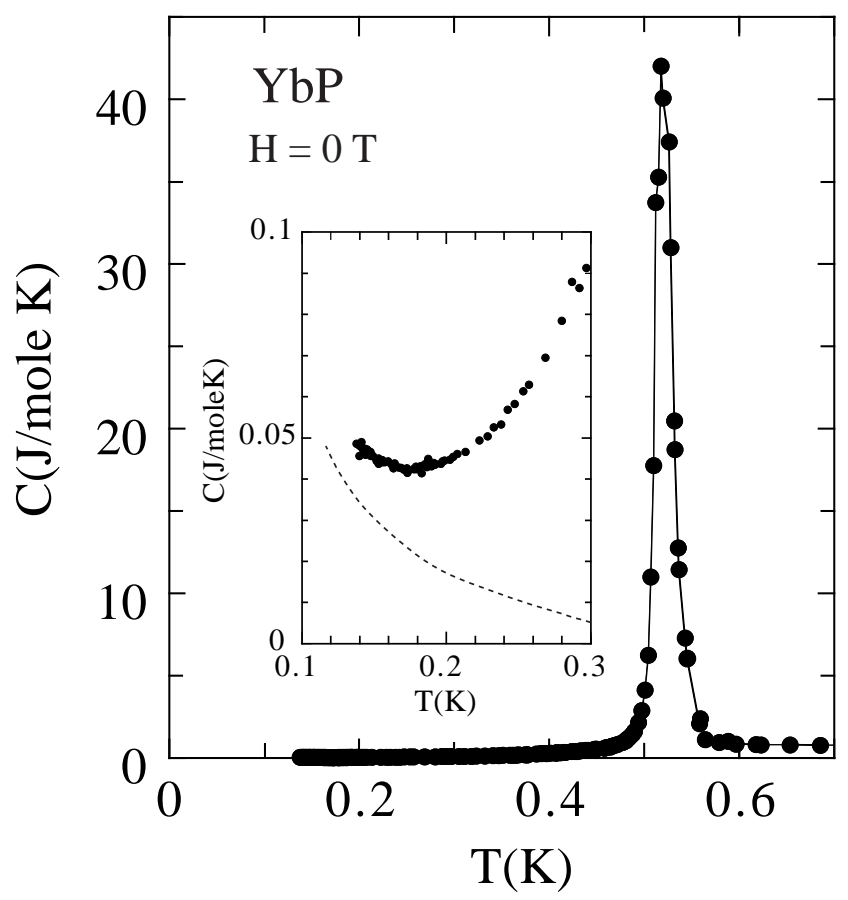

Fig. 1. Temperature dependence of the specific heat of $\mathrm{YbP}$ in zero field. A sharp peak near $T=0.5 \mathrm{~K}$ indicates the antiferromagnetic phase transition. The inset shows the low temperature part. The dotted line indicates the nuclear contribution of the specific heat which is estimated from the hyperfine field derived from the measurement of ${ }^{170} \mathrm{Yb}$ Mössbauer effect.

$=1: 1.1$ in a closed quartz ampoule. The elements were heated for 2 weeks up to $380^{\circ} \mathrm{C}$, and annealed for 1 week at $700{ }^{\circ} \mathrm{C}$. The neutron diffraction experiments were performed on this powder sample by Keller et al. ${ }^{9)}$ and revealed the average phosphorus content of $x=0.99(1)$ per formula $\mathrm{YbX}_{x}$ indicating almost ideal stoichiometry. The powder material of $\mathrm{YbP}$ was then pressed into a hard pellet and sealed in tungsten crucible. Finally, the crucible was heated up to above $2500{ }^{\circ} \mathrm{C}$ and kept at this temperature for 72 hours. A single crystal of about $5 \times 5 \times 5 \mathrm{~mm}^{3}$ was obtained in this way. X-raydiffraction patterns showed a NaCl-type single phase for this sample with the lattice parameter value of $5.542 \AA$ at room temperature. Chemical analysis yielded the value of 1:1.00 \pm 0.01 for the atomic ratio between $\mathrm{Yb}$ and $\mathrm{P}$ consistent with the neutron-diffraction results mentioned above. We measured the specific heat by means of a conventional adiabatic heat pulse method in the temperature range of $0.13-12 \mathrm{~K}$ generating by a ${ }^{3} \mathrm{He}-{ }^{4} \mathrm{He}$ dilution refrigerator. An external magnetic field up to $16 \mathrm{~T}$ was applied using a superconducting magnet. A $\mathrm{RuO}_{2}$ resistance thermometer is located in the compensated field in order to avoid the field dependence of the resistance.

\section{$\S 3 . \quad$ Results and Discussion}

The temperature dependence of the specific heat $C(T)$ in zero field is shown in Fig. 1. The peak at $0.53 \mathrm{~K}$ is due to the antiferromagnetic phase transition. The peak is sharp and the peak value is three times larger than (a)

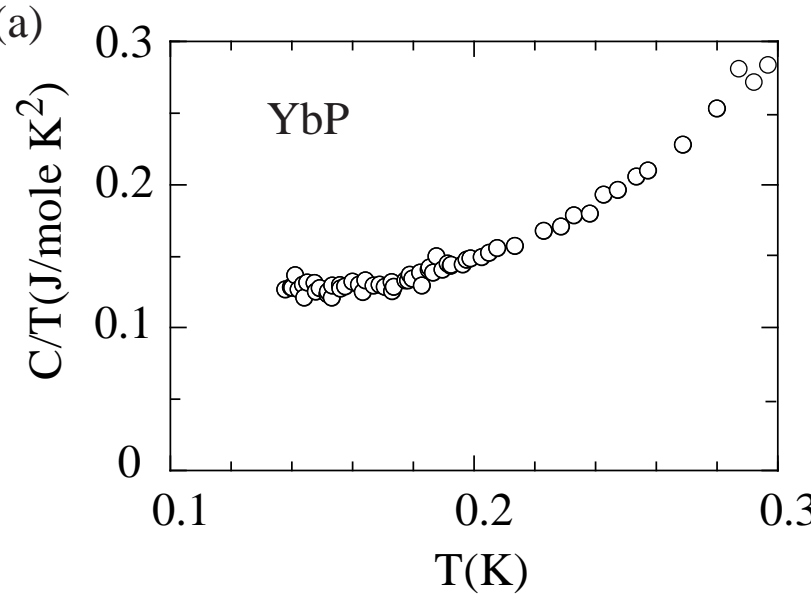

(b)

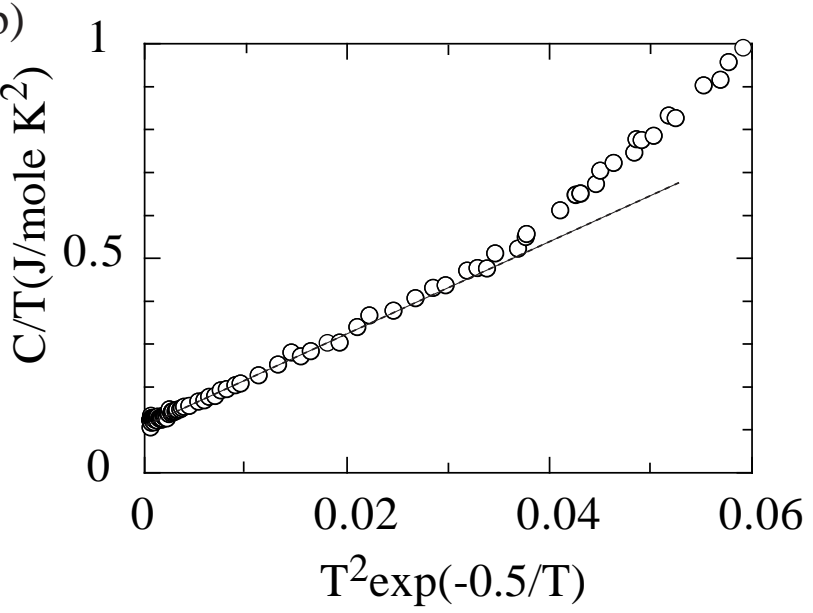

Fig. 2. (a) $C / T$ vs. $T$ plot of $\mathrm{YbP}$. $C / T$ shows an almost constant value of about $130 \mathrm{~mJ} / \mathrm{moleK}^{2}$ below $170 \mathrm{mK}$. (b) $C / T$ vs. $T^{2} \exp (-0.5 / T)$ plot of YbP. The dashed line represents "leastsquares" fit using Eq.(1) in the temperature range from $138 \mathrm{mK}$ and $350 \mathrm{mK}$.

that in the previous result, indicating a good quality of our single crystal sample. ${ }^{10}$ The increase of the specific heat below $170 \mathrm{mK}$ is attributed to the nuclear Zeeman effect of $\mathrm{Yb}$ nuclei suffering an exchange field in the antiferromagnetic state.

The specific heat of $\mathrm{YbP}$ contains a phonon part $C_{\mathrm{p}}$ (proportional to $T^{3}$, which is important only at higher temperature and thus is neglected in the following analysis), a nuclear part $C_{\mathrm{n}}$ an electronic part $C_{\mathrm{e}}$ and a magnetic part $C_{\mathrm{m}}$. We estimated the nuclear contribution of the specific heat from the hyperfine field $\left(H_{\mathrm{hf}}=93 \mathrm{~T}\right)$ derived from the measurements of the ${ }^{170} \mathrm{Yb}$ Mösbauer effect as denoted in dotted line in Fig. $1{ }^{14)}$ By subtracting the nuclear contribution from the total specific heat, the electronic specific heat $C(T)\left(=C_{\mathrm{e}}+C_{\mathrm{m}}\right)$ was obtained and plotted in Fig. 2(a). $C / T$ seems to approach constant value around $130 \mathrm{~mJ} / \mathrm{moleK}^{2}$ below $170 \mathrm{mK}$. @

Next we consider the contribution from the antiferromagnetic spin wave excitation to the specific heat $C_{\mathrm{m}}$. We could not fit the experimental result if we assumed that antiferromagnetic spin wave dispersion does not have a gap and $C_{\mathrm{m}}$ is expressed as $C_{\mathrm{m}} \sim T^{3}$. Thus, 

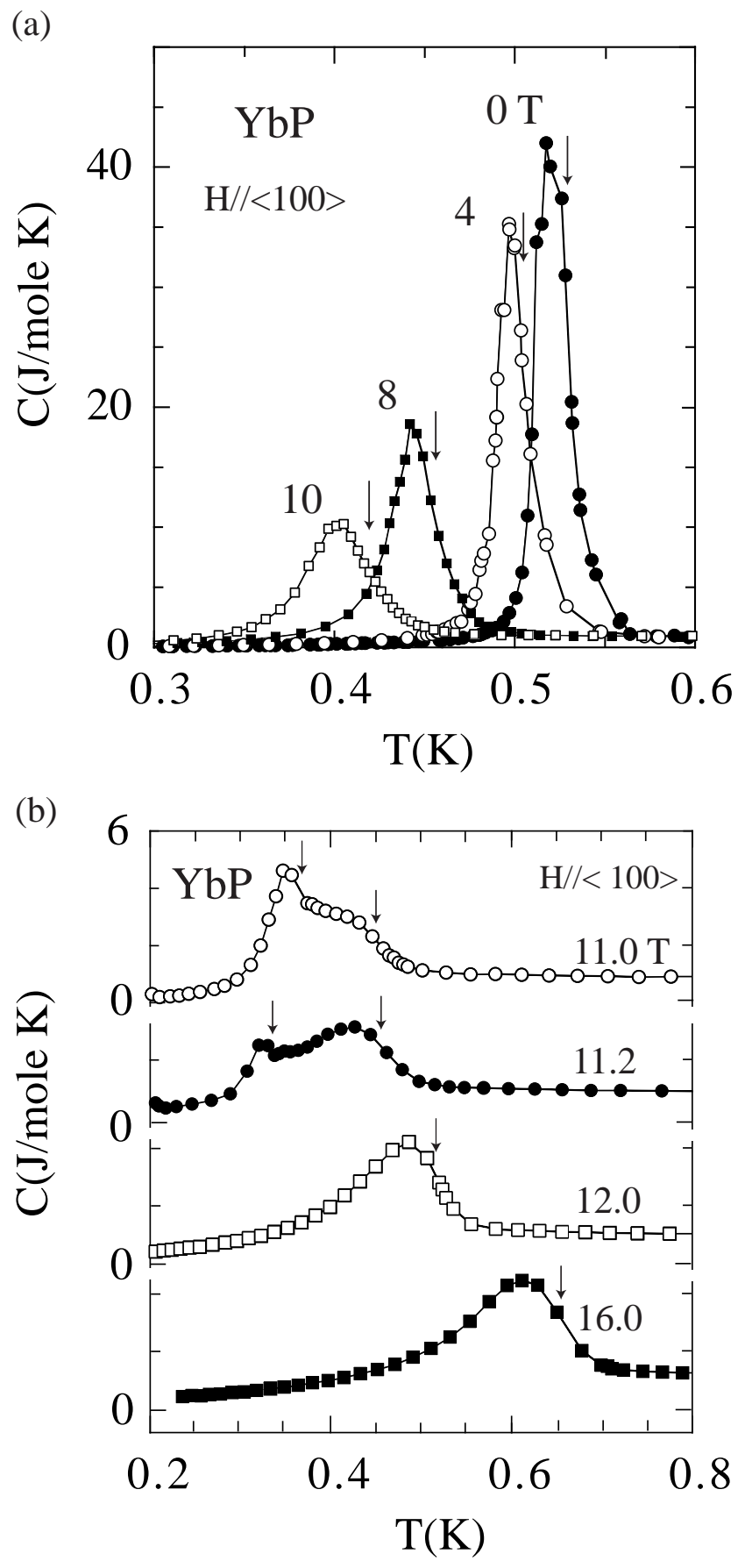

Fig. 3. Specific heat of $\mathrm{YbP}$ in magnetic ifelds up to 16 T. $C(T)$ shows a single discontinuity at $T_{\mathrm{N}}$ below $10 \mathrm{~T}$ (a). A two peak structure appear at $11.0 \mathrm{~T}$ and $11.2 \mathrm{~T}(\mathrm{~b})$. The transition temperatures are denoted by arrows.

it is suggested that the antiferromagnetic spin wave excitation of YbP has a gap similar to that of YbAs. In YbAs, Dönni et al. have performed an inelastic neutron scattering experiment and reported that one of two magnon branches has a $k$-linear dispersion near the magnetic zone center with an energy gap of $0.08 \mathrm{meV} .{ }^{15)} \mathrm{We}$ fit the measured specific heat data using the expression

$$
C=C_{\mathrm{e}}+C_{\mathrm{m}}=\gamma T+\delta T^{3} \exp (-\Delta / T)
$$

in the temperature range from $138 \mathrm{mK}$ to $350 \mathrm{mK}$. The

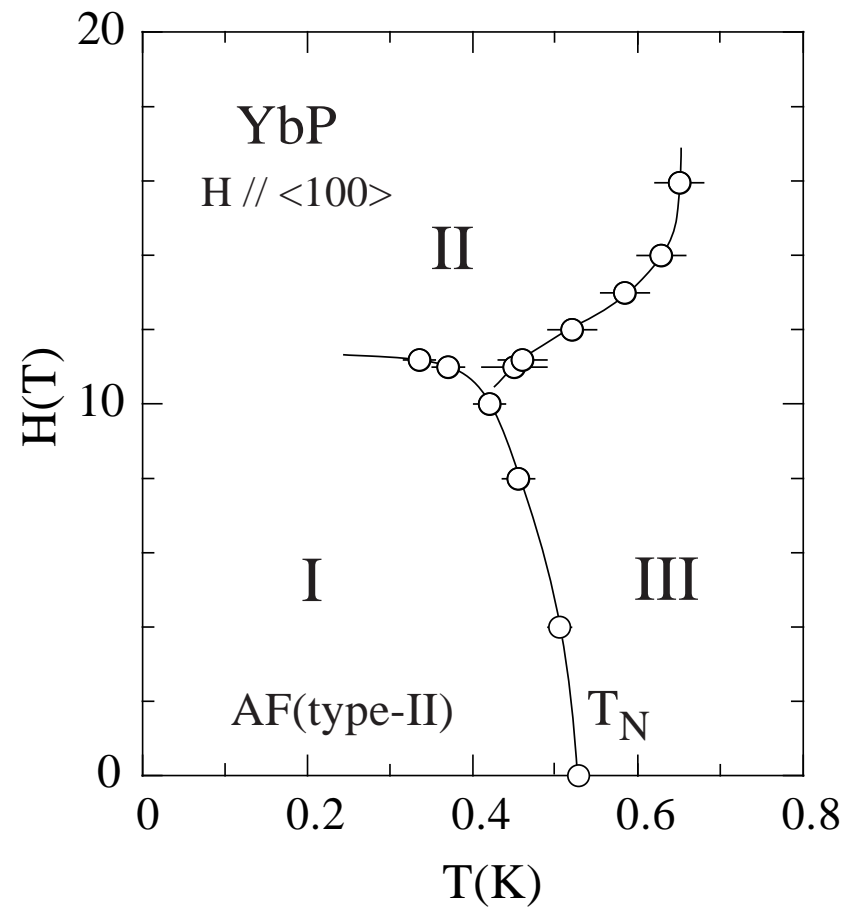

Fig. 4. Magnetic phase diagram of YbP for the magnetic field along $\langle 100\rangle$ direction obtained from the specific heat measurements

first term indicates the electronic specific heat $C_{\mathrm{e}}$. The second term indicates the antiferromagnetic magnon contribution to the specific heat $C_{\mathrm{m}}$ and we use a same expression that Sakon et al. used in the analysis of the specific heat of YbAs. ${ }^{11)}$ The energy gap $\Delta$ and the coefficient of the electronic specific heat $\gamma$ are estimated to be $0.5 \mathrm{~K}$ and $115 \mathrm{~mJ} / \mathrm{moleK}^{2}$ mole, respectively. This $\gamma$ value is compatible with the constant $C / T$ value observed below $170 \mathrm{mK}$.

We calculated $C_{\mathrm{m}}$ with eq. (1) using obtained parameters and found that $C_{\mathrm{m}}$ is only $6 \%$ of the specific heat $C(T)\left(=C_{\mathrm{e}}+C_{\mathrm{m}}\right)$ at $150 \mathrm{mK}$. Thus, about $90 \%$ of the constant $C / T$ observed below $170 \mathrm{mK}$ comes from the electronic specific heat $C_{\mathrm{e}} / T$. From these results, we conclude that the $\gamma$ value is about $120 \mathrm{~mJ} / \mathrm{moleK}^{2}$ in YbP. Note that a large $\gamma$ value of $270 \mathrm{~mJ} / \mathrm{moleK}^{2}$ was also reported for YbAs. ${ }^{11)}$

The $\gamma$ values of $\mathrm{YbP}$ and $\mathrm{YbAs}$ are much larger than those of normal metals, indicating the formation of heavy fermion state in both systems. The anomalous physical properties in $\mathrm{Yb}$ monopnictides are usually interpreted in terms of the competition between the Kondo effect and RKKY interaction. In this mechanism, the Kondo effect was due to a large mixing between $4 f \Gamma_{6}$ hole state of the $\mathrm{Yb}^{3+}$ ion and the occupied $p-\Gamma_{6}$ valence-hole states. Thus, the existence of $p-\Gamma_{6}$ holes in $\mathrm{YbXp}$ is the prerequisite for the Kondo effect.

Usually, for a metallic heavy Fermion compound, the concentration of conduction electrons is nearly the same order of magnetic ions. However, the problem is that the total hole carrier numbers in $\mathrm{YbAs}$ and $\mathrm{YbP}$ are 


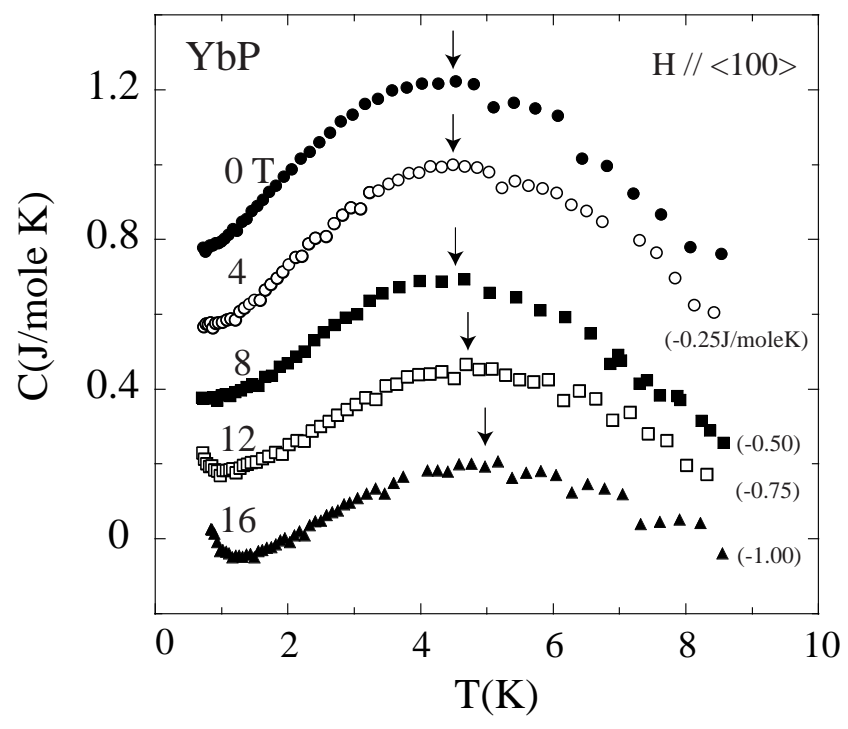

Fig. 5. Temperature dependence of the specific heat of $\mathrm{YbP}$ at higher temperatures in the magnetic fields up to $16 \mathrm{~T}$. The curves in higher fields are sifted downwards for the sake of clarity.

the order of 0.01 per $\mathrm{Yb}$ atom. Moreover $p-\Gamma_{6}$ hole was not observed in the dHvA experiment on YbAs. ${ }^{16)}$ Sakon et al. and Hashi et al. proposed a conjecture that heavy Fermion state in $\mathrm{Yb}$ monopnictides may be realized by antiferromangetic correlations between the localized moments of the $\mathrm{Yb}$ ion due to the exchange interaction. ${ }^{11,17,18)}$ In this mechanism, the magnitude of the localized moments is reduced by this antiferromagnetic correlations and the Kondo type screening of the localized moments just help the mutual reduction of the localized moments. On the different point of view, a charge dipolar model was recently proposed and used to explain the experimental results of $\mathrm{Yb}$ monopnictides by Kasuya et al. ${ }^{19)}$ At present, it is impossible to discuss the validity of above scenarios within the present experimental results because these theoretical predictions are limited within qualitative considerations. In order to clarify the heavy fermion state in $\mathrm{Yb}$ monopnictides, further theoretical investigations and quantiative comparison with the theories and experimental results are needed.

Fig.3 (a) and (b) show the temperature dependence of the specific heat of $\mathrm{YbP}$ under magnetic fields between $0<H<10 \mathrm{~T}$ and $11<H<16 \mathrm{~T}$, respectively, applied along the $\langle 100\rangle$ direction. Below $10 \mathrm{~T}$, a single peak appears in $C(T)$ at the Néel temperature $T_{\mathrm{N}}$. With increasing $H$, the peak loses intensity and the position shifts to lower temperature. It is noted that $C(T)$ curve shows two peaks in the external fields of $11.0 \mathrm{~T}$ and 11.2 $\mathrm{T}$ as illustrated in Fig. 3 (b), indicating that there clearly exists an additional phase transition in the ordered phase. We defined the transition temperature such that the entropy is conserved, that is, entropy balance. In this way, the Néel temperature of our YbP sample is estimated to be $530 \mathrm{mK}$ at zero magnetic field. This value is smaller than $T_{\mathrm{N}}=660 \mathrm{mK}$ determined by neutron diffraction experiments for the prereacted powder sam-

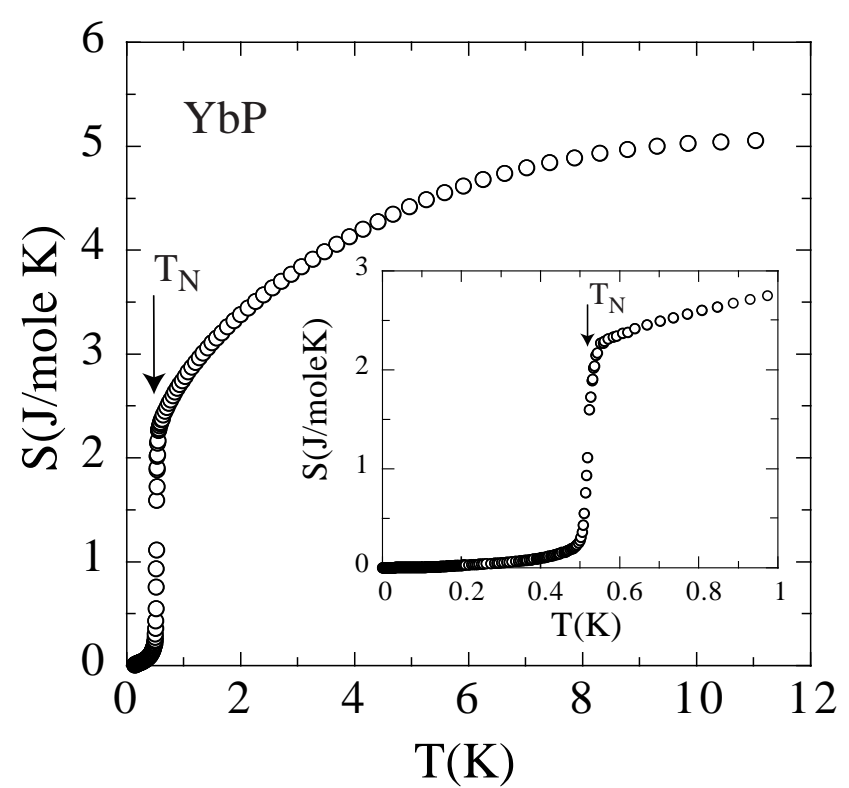

Fig. 6. Temperature dependence of the entropy of $\mathrm{YbP}$ in zero field.

ple. ${ }^{9)}$ It may be attributed to either sample dependence of difference in the definition of transition temperature. With these transition temperatures, we have determined a magnetic phase diagram as shown in Fig. 4. Here, the phases are referred to as I, II and III. The phase I is the antiferromagnetic ordered state which is previously reported. The phase II is revealed for the first time in this measurement. The phase III is a paramagnetic phase. As the field strength increases, the transition temperature from the phase I to III shifts to lower temperature. On the other hand, the transition temperature from II to III increases with the magnetic field.

In Ce monopnictides such as $\mathrm{CeSb}, \mathrm{CeP}$ and $\mathrm{CeAs}$, complicated magnetic phase diagrams or multi step meta-magnetic transitions were observed. ${ }^{20)}$ Neutron scattering experiments revealed that complicated magnetic structures appear in the magnetic field in $\mathrm{Ce}$ monopnictides. ${ }^{21,22)}$ Many theoretical studies have been done in order to explain the unusual magnetic properties of Ce monopnictides. ${ }^{20}$ ) Note that the magnetic phase diagram of YbP has some similarity with that of Ce monopnictide. It is interesting to perform neutron scattering experiment or magnetization measurement on $\mathrm{YbP}$ and compare magnetic properties with those of $\mathrm{Ce}$ monopnictides.

Fig.5 shows the temperature dependence of the specific heat in high temperature region. For the sake of clarity, we omit the data below $0.8 \mathrm{~K}$ and the curves in the higher fields are shifted downwards by the values in parentheses. A broad maximum exists around 4 $\mathrm{K}$ in $C(T)$ curve, which is consistent with the previous reports. ${ }^{10,23)}$ This peak was considered to be a Kondo peak with $T_{\mathrm{K}}$ of about $5.7 \mathrm{~K}^{23)}$ Recently the results of the inelastic neutron scattering experiment showed that short range antiferromagnetic correlations in $\mathrm{YbX}_{\mathrm{p}}$ be- 
gin to develop below about $20 \mathrm{~K}$ far above $\mathrm{T}_{\mathrm{N}}{ }^{24)}$ and then the broad specific heat maximum may be related to this antiferromagnetic correlation. The position of the broad maximum shows no distinct change in lower fields and slightly sifts to higher temperatures with increasing the field above $8 \mathrm{~T}$. This feature can not be explained in terms of antiferromagnetic correlations. We suggest that a short range ferromagnetic correlation is induced in the magnetic field above $10 \mathrm{~T}$ and this correlation may be related to the appearance of the phase II above $10 \mathrm{~T}$.

We have also estimated the entropy by integrating $C / T$ curve with respect to $T$ as shown in Fig. 6. To obtain the entropy $S$, we extract the nuclear part of the specific heat, $C_{\mathrm{n}}$ obtained from the Mössbauer measurement as described above. We extrapolate $C / T$ curve below 138 $\mathrm{mK}$ to zero temperature using a relation $C=\gamma^{\prime} T$ with $\gamma^{\prime}=130 \mathrm{~mJ} / \mathrm{moleK}^{2}$. The appearance of a sharp peak in $C(T)$ and sudden change in $S(T)$ at $T_{\mathrm{N}}$ suggest that a first-order phase transition occurs at this temperature. At $T_{\mathrm{N}}, S$ reaches $40 \%$ of $R \ln 2=5.76 \mathrm{~J} / \mathrm{moleK}$, the expected entropy of a $\Gamma_{6}$ doublet ground state. This $S$ value is two times larger than that reported in Ref.10 in which the Néel temperature of $\mathrm{YbP}$ was determined to be $0.41 \mathrm{~K}$. The sample quality may be responsible for this difference. In this work, we used a high quality single crystal sample that shows the higher Néel temperature $(0.53 \mathrm{~K})$ and a larger transition peak. Thus the estimation of the entropy in the present study is more reliable. The total entropy $R \ln 2$ is recovered if the temperature is raised up to the temperature much above $10 \mathrm{~K}$, that is, more than an order of magnitude above the magnetic ordering temperature. This feature resembles to that of the dense Kondo Cerium compounds and again suggests the formation of heavy Fermion state in YbP.

\section{§4. Conclusion}

In conclusion, we have measured the low temperature specific heat of $\mathrm{YbP}$ up to $16 \mathrm{~T}$ using a stoichiometric single crystal sample. The $\gamma$ value and the magnetic phase diagram in $\mathrm{YbP}$ were determined. The large $\gamma$ value and the reduced entropy at the magnetic ordering temperature suggest the formation of heavy Fermion state in YbP. A new magnetic phase was observed under the high magnetic field of $\mathrm{YbP}$ and the magnetic phase diagram has some similarity comparing with that of Ce monopnictides. This suggests that some interesting magnetic phenomena similar to those observed for Ce monopnictides could also be expected for $\mathrm{Yb}$ monopnictides.

1) A. Oyamada: Dr. Thesis, Tohoku University, 1991.

2) D. X. Li: Dr. Thesis, Tohoku University, 1994.

3) A. Dönni, A. Furrer, P. Fischer, F. Hulliger and P. Wachter: Physica B 171 (1991) 353.

4) M. Kohgi, K. Ohayama, A. Oyamada, T. Suzuki and M. Arai: Physica B 163 (1990) 625.

5) K. Ohyama: Dr. Thesis, Tohoku University, 1992.

6) A. Hasegawa: J. Phys. Soc. Jpn. 246 (1985) 77.

7) H. Harima and T. Kasuya: J. Magn. Magn. Mater. 52 (1985) 370

8) A. Dönni, A. Furrer, P. Fischer, F. Hulliger, P. Wachter and H. R. Ott: J. Magn. Magn. Mater. 90-91 (1990) 143.

9) L. Keller, P. Fischer, A. Furrer, A. Dönni, D. X. Li and T.
Suzuki: Physica B 186-188 (1993) 553.

10) H. R. Ott, H. Rudigier and F. Hulliger: Solid State Commum. 55 (1985) 113.

11) T. Sakon, N. Sato, A. Oyamada, N. Takeda, T. Suzuki and T. Komatsubara: J. Phys. Soc. Jpn. 61 (1992) 2209.

12) A. Oyamada, C. Ayache, T. Suzuki, J. Rossat-Mignod and T. Kasuya: J. Magn. Magn. Mater. 90-91 (1990) 443.

13) D. X. Li, K. Sumiyama, K. Suzuki, A. Oyamada, K. Hashi and T. Suzuki: Phys. Rev. B 57 (1998) 12036.

14) G. Le Bras, P. Bonville, J. A. Hodges, P. Imbert, G. Polatsek, P. Fischer, L. Keller, A. Donni, M. Kohgi and T. Suzuki: Physica B 190 (1993) 333.

15) A. Dönni, A. Furrer, P. Fischer, F. Hulliger and S. M. Hayden: J. Phys.: Condens. Matter 4 (1992) 4283.

16) N. Takeda: Dr. Thesis, Tohoku University, 1991

17) K. Hashi, A. Oyamada, S. Maegawa, T. Goto, D. X. Li and T. Suzuki. 67 (1998) 4260.

18) K.Ueda, Progress of the Theoretical Physics, Supplement 108 (1992) 133.

19) T. Kasuya and D. X. Li: J. Phys. Soc. Jpn. 66 (1997) 1587.

20) For a review, see T. Kasuya, Physica B 215 (1995) 88.

21) M.Kohgi, T. Osakabe, K. Iwasa, J. M. Mignot, I. N. Goncharenko, Y. Okayama, H. Takahashi, N. Mori, Y. Haga, and T. Suzuki: J. Phys. Soc. Jpn 65 (1996) Supplement. B 99.

22) J. Rossat-Mignod, J. M. Effantin, P.Burlet, T.Chattopahyay, L.P.Regnault. H. Bartholin, C. Vettier, P. Vogt, D. Ravot and J. C. Achart: J. Magn. Magn. Mater. 52 (1985) 111.

23) S. Takagi, A. Oyamada and T. Kasuya: J. Phys. Soc. Jpn 57 (1988) 1456.

24) K. Ohoyama, T. Osakabe, A. Dönni, M. Kohgi, A. Oayamada, T. Suzuki: Physica B 213-214 (1995) 119. 